\title{
EDITORIAL
}

\section{Enterprise Risk Management}

\author{
Alexander J. McNeil* \\ Department of Actuarial Mathematics \& Statistics, Heriot-Watt University, Edinburgh, UK \\ Maxwell Institute for Mathematical Sciences, Edinburgh
}

This Special Issue is devoted to Enterprise Risk Management (ERM), an active interdisciplinary research area that is an increasingly important part of modern actuarial science. Arguably, actuaries have always been risk managers, applying their skills to measure and mitigate the risks of future events. However, the development of modern regulation in financial services - Solvency II for European insurers and the Basel Accords in banking - has led to the development of a more clearly defined profile for enterprise risk managers. The worldwide actuarial profession has responded to this by creating the CERA (Chartered Enterprise Risk Actuary) qualification and defining the skill set that enterprise risk actuaries require. In parallel with the professional focus on Enterprise Risk Management, the volume of important research into the open problems of ERM increases year on year.

To some extent methodological research in ERM currently falls between stools in that, depending on topic, an ERM research paper might be suitable for a finance journal, an economics journal, a statistics or econometrics journal, a financial mathematics journal or an actuarial journal. Specialist risk management journals have appeared but are still establishing themselves as outlets for academic research while serving a mixed community of university and industry-based researchers.

This Special Issue aims to underscore the importance that the Annals of Actuarial Science places on research in ERM. It contains a small sampling of some of the different research themes that fall under the general heading of ERM. The thread that connects the papers together is the study of dependent risks and extreme risks.

In the paper by Merz, Wüthrich and Hashorva on "Dependence modelling in multivariate claims run-off triangles", the authors revisit the classical claims reserving problem and add to the growing literature on modelling dependencies between different accounting years and different cells in runoff triangles. Using a multivariate log-normal model they are able to compute closed form solutions for claims reserves and quantify the uncertainty associated with claims reserves estimates.

Their results give new insights into the general problem of claims reserving and address an open issue in the implementation of Solvency 2. Indeed they suggest that the way that run-off triangle correlations are currently specified in both Solvency 2 and the Swiss Solvency Test (SST) is likely to underestimate the prediction uncertainty for future claims.

*Correspondence to: Professor A. McNeil, Department of Actuarial Mathematics \& Statistics, Heriot-Watt University, Colin Maclaurin Building, EDINBURGH, EH14 4AS, United Kingdom. Tel: +44 (0)131 4513230. Fax: +44 (0)131 451 3249. E-mail: A.J.McNeil@hw.ac.uk 
Dependence modelling in risk management has proved, in general, to be a very fertile area for research. The financial crisis has served to underline the importance of topics like systematic risk, diversifiability of risks (or the lack thereof) and heavy tails.

These ideas come together in the contribution by Mainik and Embrechts entitled "Diversification in heavy-tailed portfolios: properties and pitfalls". In particular, if risk is measured using the nonsubadditive VaR measure, what kind of situations lead to superadditivity of the portfolio risk with respect to its component risks and the ensuing loss of diversification that this implies? The authors address this question in the context of multivariate regularly-varying risk factor distributions, an important class of heavy-tailed models. They derive very general results, which they illustrate with a number of insightful special cases. It turns out that the issue of diversification is not straightforward and is not only influenced by the heaviness of distributional tails but also the extent to which losses and gains compensate each other.

Extreme value modelling ideas also appear in the paper of Ganegoda and Evans on "A scaling model for severity of operational losses using generalized additive models for location scale and shape (GAMLSS)." As the title indicates, the sphere of application for their work is operational risk where one of the greatest problems to be overcome is the sparseness of data for individual banks. In Basel II banks are required to combine their internal loss data with external data, but there are all kinds of practical issues involved in the problem of combining the two data sources. In particular external data are prone to a number of inherent biases due, for example, to the use of different thresholds for data inclusion at different banks (reporting bias) and the very different scales of losses at banks of different sizes (scale bias).

The authors use a combination of an extreme value approach and a generalized additive model for scale to overcome these two biases and they validate their approach using data from the US banking industry. Their work is an impressive piece of statistical modelling that shows that, with the right degree of care, sensible results may be extracted from pooled operational data sources.

The final paper in this issue is a contribution by Hürlimann on the topic of "Economic Capital Modelling for the Motor Third Party Liability (MPTL) man-made catastrophe risk." In this general insurance context extreme value modelling is also important. The author examines the simple approach applied in the QIS5 "quantitative impact study" to compute economic capital for MPTL cat risk. In QIS5 the total insurance loss takes the form of a single claim and the author argues that it would be more appropriate to model it with a dynamic model with Poisson occurrence of claims and Pareto severities. He shows that, under certain conditions, the QIS5 approach can be justified by an asymptotic analysis of the preferred dynamic model. Moreover he derives new formulas for the case in which limits of cover are applied to the contract.

These four papers are all motivated, directly or indirectly, by the regulatory environment and, as long as this remains in flux, it is to be expected that enterprise risk management will be a fertile research area. It is to be hoped that more important contributions in the field will be published in this journal in the future. 